\title{
El conflicto epistémico de la pulsión
}

\section{Drive's epistemic conflict}

\author{
ORIARD-VALLE, Michel $\dagger^{*} \&$ JUÁREZ-CAMPUSANO, Yara Suhan \\ Universidad Tecnológica de México (UNITEC), Campus Querétaro, Av. 5 de Febrero 1412, San Pablo, 76130 Santiago de \\ Querétaro, Qro.
}

ID $1^{\text {er }}$ Autor: Michel, Oriard-Valle / ORC ID: 0000-0002-1186-0941, Researcher ID Thomson: S-5919-2018, CVU CONACYT ID: 781986

ID $1^{\text {er }}$ Coautor: Yara Suhan, Juárez-Campusano / ORC ID: 0000-0003-0886-5466, CVU CONACYT ID: 710264

DOI: $10.35429 / J P D L .2019 .14 .5 .13 .19$

Recibido 10 de Enero, 2019; Aceptado 30 Marzo, 2019

\begin{abstract}
Resumen
En el presente artículo se analizó la validez epistemológica del término pulsión (trieb) en psicoanálisis a partir del contraste entre su pretendido origen biológico en la teoría de Sigmund Freud y los resultados actuales de la genética. Para Freud la cultura resultó de un momento de la evolución humana en que fue imprescindible crear alianzas entre los primitivos para afrontar los peligros naturales. Por lo que su principal propósito es protegernos de la naturaleza y regular los vínculos sociales. En este orden de ideas es necesario suponer que previo a la cultura el humano ya tenía pulsiones y debido al sacrificio pulsional que impone la cultura es posible convivir en comunidad. Dado que las pulsiones, en esta teoría, son la fuente del sufrimiento psíquico y los recursos empleados para defenderse de él son culturales, el modo en que estas sean consideradas repercute sobre el fundamento de las intervenciones clínicas y las finalidades del tratamiento psicoanalítico. Actualmente la genética pone en tela de juicio la existencia autónoma de una naturaleza humana inalterable por la cultura. En otras palabras, los estudios epigenéticos, la reproducción asistida, la clonación, entre otros, cuestionan seriamente que haya una supuesta naturaleza biológica inmutable en el humano.
\end{abstract}

Psicoanálisis, Pulsión, Epistemología

\begin{abstract}
This article analyzed the epistemological validity of the term drive (trieb) in psychoanalysis by contrasting its supposed biological origin in Sigmund Freud's theory with recent genetic results. Culture, for Freud, appeared in a moment of human evolution when it became necessary for our ancestors to form alliances in order to face natural hazards. Therefore culture's main purpose is to protect us from nature and to regulate social relationships. Consequently it is implied that drives formed part of human's nature before culture existed and due to drive's sacrifice that culture demands it's possible to live in society. Given that drives, in Freud's theory, are the source of psychic suffering and the resources used to defend against it are cultural, the way in which these are considered have repercussions on the basis of clinical interventions and the aims of psychoanalytical treatment. At present, genetics questions the idea that human nature cannot be modified by culture. In other words, epigenetic studies, assisted reproduction, cloning, among others, seriously query the idea of an unchangeable human nature.
\end{abstract}

Psychoanalysis, Drive, Epistemology

Citación: ORIARD-VALLE, Michel \& JUÁREZ-CAMPUSANO, Yara Suhan. El conflicto epistémico de la pulsión. Revista de Filosofía y Cotidianidad. 2019, 5-14: 13-19

\footnotetext{
* Correspondencia del Autor (orvami @ outlook.com)

$\dagger$ Investigador contribuyendo como primer autor.
} 


\section{Introducción}

En la teoría de Sigmund Freud, la pulsión (trieb) es uno de sus conceptos cruciales para la metapsicología dado que posibilita el entendimiento sobre la causa del sufrimiento psíquico y cómo intervenir sobre él. A la pulsión se le reconocen diferentes propiedades; entre ellas una naturaleza biológica como su fundamento. Vale decir que el quid de la pulsión sería esta propiedad y en la medida que uno la comprendiese su intervención será más pertinente para abordar el malestar.

El objetivo del presente trabajo es contrastar la pretendida naturaleza biológica de la pulsión con la biología contemporánea para analizar su validez epistémica. En tal sentido se procede a señalar la importancia del fundamento conceptual, a revisar las características que posee la pulsión en la teoría de Freud, exponer qué se entiende por energía y naturaleza en biología y al análisis de los resultados.

\section{Fundamento teórico}

El modo en que un elemento conceptual funciona en una teoría y cómo es entendido repercute sobre su operatividad en la práctica (Badiou, 2009; Koyré, 2007). Vale decir que ciertos fenómenos involucrados en la estructura del problema que determinado modelo teórico aborda para su resolución tendrán valor existencial por su fundamentación. En la medida que se carezca de esta, su pertinencia y eficiencia será menor para solucionar los problemas específicos a los que se dedica. La coherencia conceptual se ve influida por su relación con otros sistemas teóricos (Subaş1, 2019). En este orden de ideas, mientras más aislado permanezca un modelo teórico de los desarrollos de otros menor será su capacidad explicativa (Eidelsztein, 2008).

La contrastación epistémica clarifica y cambia la compresión de los fenómenos (Kreiss, 2019; Van Damme et al., 2019), permite reconocer cuando la actividad de los practicantes de una disciplina está orientada por la ortodoxia (Ballantyne, 2019) y contribuye a la orientación de investigaciones científicas; así como a los ejercicios interdisciplinarios (Bachelard, 2010; Lakatos, 2007).
Sobre la base de las consideraciones anteriores, el contraste epistémico entre el psicoanálisis propuesto por Freud y la biología respecto al término pulsión esclarece la pertinencia y alcance del fundamento para sus consecuencias clínicas.

\section{La pulsión y su carácter biológico en la teoría de Freud}

En la teoría de Sigmund Freud la pulsión (trieb) es un concepto limítrofe entre lo psíquico y lo somático dado que su procedencia es orgánica y endógena al cuerpo y sus efectos son psíquicos. Particularmente en las representaciones cuya vicisitud pulsional produce sufrimiento (Freud, 2016f). En otras palabras, las intervenciones del analista consisten en colegir el material interpretable del tratamiento psicoanalítico; por ejemplo: representaciones determinadas por la represión (verdrängung) cuya vicisitud pulsional da cuenta de estas como síntoma. Las pulsiones son consideras un tipo de energía, diferenciada del instinto y de los estímulos externos al aparato psíquico, a manera de fuente para los síntomas.

De manera general, las pulsiones cuentan con cuatro características fundamentales (Freud, 2010b). Tienen:

1. Un factor motor, sobreentendido como su suma de fuerza, es decir esfuerzo (Drang)

2. Una meta (Ziel) que implica su satisfacción a través de pensamientos o acciones.

3. Un objeto (Objekt) gracias al cual se realiza la satisfacción. Este objeto puede ser solo una representación o una representación homologada con un objeto externo al psiquismo.

4. Un proceso interno y somático que es su fuente (Quelle).

Freud reconoció diferentes nominaciones para las pulsiones. Por ejemplo, pulsiones yoicas, sexuales, de vida y de muerte. Sin embargo, siempre les atribuyó una naturaleza biológica, aunque dudaba si preponderantemente eran propiedades químicas o físicas. Así excluía estrictamente de su consideración a las investigaciones psicológicas para conocer a las pulsiones y quedaba reservada su intelección para la biología, particularmente la fisiología. 
Consecuentemente los síntomas serían manifestaciones de las pulsiones y una vía indirecta para estudiarlas.

Otro aspecto en esta teoría es que las pulsiones tienen como fin último conducir a un estado inerte que es supuesto como originario (Freud, 2010b, 2016g). El planteamiento conlleva que las pulsiones, debido a su pretendida naturaleza biológica, son las responsables de la tendencia en un organismo hacia su muerte.

Una crítica al asumir que la pulsión se comportaría como un tipo de energía -por ende, podría ser abordada desde la fisiología- es que la teoría de Freud no contempla que al tratarla como un tipo de energía debería ser posible que esta se transforme en otra; o sea que la pulsión se transforme en energía calorífica (Krymkiewicz, 2015; Swanson, 1977). Otra crítica consiste en que las características que Freud atribuyó a la pulsión cumplen como analogía expositiva, mas no propiamente como energía según las leyes de la física clásica.

Ejemplo de ello es la imprecisión de una cantidad fija de energía durante las asociaciones de representaciones. Recordando que el soporte material de las representaciones en la teoría de Freud es la actividad neural (Kandel, 2007). Por otro lado, la asunción de que la "naturaleza" biológica de la pulsión prescribe ultimadamente que todo ser vivo aspire -sin saberlo a la muerteremite más bien a la filosofía helénica (Torri, 2019).

Swanson (1977) señaló que tratar a la pulsión como un tipo de energía es incorrecto. No obstante, igual indicó que los modelos teóricos en biología parecieran los más acertados para intentar comprender los presupuestos que orientaron las argumentaciones de Freud sobre la dinámica pulsional.

Incluso Freud propuso que las pulsiones son las responsables de que el sistema nervioso lleve a cabo actividades complejas para transformar al medio y entonces cumplir con su satisfacción. Este aspecto Freud lo utilizó como argumento de la evolución en nuestra especie frente a los peligros de la naturaleza y el desarrollo cultural. Por lo tanto es necesario remitir al carácter natural y biológico que Freud reconoció a las pulsiones en contraste con la cultura.
Ideológicamente la diferencia entre naturaleza y cultura se estima así: entendiendo por la primera todo aquello que es, o está, sin intervención humana. Mientras que todo lo cultural sería artificial, o secundario a lo natural, vale decir cualquier invención humana; como las normas y valores. Ante esto se plantea que hay, por un lado, naturaleza animal y, por otro, cultura humana (Geertz, 2003). Lo natural en el humano, o sea lo innato, es llamado invariante biológico (Virno, 2005). Si a esto se le agregamos la idea de pensar al humano como un animal racional, entonces, tenemos que el humano posee naturaleza y cultura, siendo la última lo que quizá, esencialmente, lo separa de otros seres.

Actualmente esta supuesta la dicotomía naturaleza-cultura no es así de clara. La genética pone en tela de juicio la existencia autónoma de una naturaleza humana inalterable por la cultura. En otras palabras, los estudios epigenéticos, la reproducción asistida, la clonación, el Proyecto Genoma Humano, entre otros, cuestionan seriamente la idea de que haya una supuesta naturaleza biológica inmutable en el humano (Corella \& Ordovas, 2017; Descola, 2012; Robles, Ramírez, \& Velásquez, 2012). Por otra parte, el concepto de cultura, como sistema de normas y valores, cambió a partir de las teorías del análisis del discurso y de las teorías sociales actuales (De la Garza Toledo, 2018; Teun A. Van Dijk, 1999; Teun Adrianus Van Dijk, 2012). Así, el invariante biológico ha sido determinado históricamente y un argumento para esta afirmación consiste en que el binomio naturaleza-cultura no tiene equivalente al exterior de las lenguas europeas (Descola, 2012).

En la teoría de Freud, la cultura es el resultado de que en un momento de la evolución humana fue imprescindible crear alianzas entre los primitivos para afrontar los peligros naturales (Freud, 2016d). De ahí que para la existencia de la cultura:

Esta sustitución del poder del individuo por el de la comunidad es el paso cultural decisivo [...] El siguiente requisito cultural es, entonces, la justicia, o sea, la seguridad de que el orden jurídico ya establecido no se quebrantará para favorecer a un individuo [...] El resultado último debe ser un derecho al que todos -al menos todos los capaces de vida comunitariahayan contribuido con el sacrificio de sus pulsiones (Freud, 2015, p. 94). 
Por lo tanto el principal propósito de la cultura es protegernos de la naturaleza y regular los vínculos sociales. Se infiere necesario suponer que previo a la existencia de la cultura el humano ya tenía pulsiones (triebe) y, debido al sacrificio pulsional que impone la cultura es posible convivir en comunidad según las normas sociales. Significa entonces que el sufrimiento psíquico es producto de nuestra cultura, pues su fuente son las pulsiones (Freud, 2010a, 2010c) y los recursos empleados para defenderse de él son culturales (Freud, 2015). De ahí que, siguiendo a Freud, “[...] la cultura yugula el peligroso gusto agresivo del individuo debilitándolo, desarmándolo, y vigilándolo mediante una instancia situada en su interior, como si fuera una guarnición militar en la ciudad conquistada" (Freud, 2015, p. 120). Por lo que el superyó (Über-Ich) no sólo es el heredero del complejo de Edipo sino el portavoz cultural de la filogenia en nuestra especie (Freud, 2015, 2016a, 2016b, 2016c, 2016e, 2016f, 2016h, 2016i, 2016j).

Dado que las pulsiones son consideradas innatas, de naturaleza biológica ya que su fuente (quelle) son procesos somáticos al interior del organismo (Freud, 2010b), su funcionamiento es disímil al de la cultura y, por lo tanto, el superyó como portavoz cultural exige cosas al individuo que contrarían a su naturaleza; vale decir, a su invariante biológico.

En la investigación y la terapia de las neurosis llegamos a hacer dos reproches al superyó del individuo: con la severidad de sus mandamientos y prohibiciones se cuida muy poco de la dicha de este, pues no tiene suficientemente en cuenta las resistencias a su obediencia, a saber, la intensidad de las pulsiones del ello y las dificultades del mundo circundante objetivo [real]. Por eso en la tarea terapéutica nos vemos precisados muy a menudo a combatir al superyó y a rebajar sus exigencias (Freud, 2015, p. 138).

\section{¿Qué se entiende por energía y naturaleza en biología?}

En biología, hablar de naturaleza resulta exhaustivo, la concepción estricta de esta puede llegar a ser ambigua y prestarse a varias interpretaciones.
Siempre que se habla de este término nos referimos particularmente a "[...] lugares $\mathrm{y}$ organismos "salvajes", que existen y se desarrollan independientes de lo humano [...]" (Klier, 2016); es decir, nos remite a configurar "algo" que no fuese elaborado por el hombre. Por supuesto, que el hombre, antes de crear, fue productor de la vida misma.

La vida es la representación, la $<$ presencia>>, de químicas pasadas, de un medio ambiente de la Tierra primitiva que, por causa de la vida, persiste en la Tierra moderna. Es la encapsulación acuosa, acotada por una membrana, del espacio tiempo. La muerte es parte de la vida, una vez que se reproduce, rescata sistemas químicos complejos $\mathrm{y}$ estructuras disipativas que surgen del equilibrio termodinámico (Margulis \& Sagan, 1996).

Por decirlo de alguna forma, la naturaleza es vida, por lo tanto, todo lo vivo tiene esta característica. En este sentido, la relación de propiedades asociadas a la pulsión termina siendo oscuro, aun forzándola sólo se puede adecuar en un modo en que ésta forma parte de algo vivo y por lo tanto natural, pero no explica el aspecto psíquico, pues "La intuición védica de que la conciencia individual es ilusoria y que cada uno de nosotros pertenece a un único sustrato primordial” (Margulis \& Sagan, 1996).

Por otro lado, si se considera a la pulsión como un tipo de energía biológica, debemos adentrarnos a la fisiología y la ecología. Desde el punto de vista de la primera, la energía se explica como el resultado de un conjunto de procesos químicos, que iniciaron y finalizaron en las células, y "[...] lo único de interés primordial para nosotros es que sentimos, pensamos y percibimos. Para el proceso fisiológico que es responsable del pensamiento y el sentido, todos los demás juegan un papel auxiliar, al menos desde el punto de vista humano" (Schrödinger, 2015).

La energía toma protagonismo a partir de la formación de una sola célula, y juega un papel distinto cuando la biología estudia las relaciones a nivel inter e intra especies más factores abióticos, tal es el caso de la ecología. 
Las formas de vida más "simples" utilizan energía metabólica (energía cinética, calórica y química) (Macalady et al., 2013) y ésta mueve todos los procesos internos y sus relaciones externas con otros individuos, si estos individuos mueren simplemente la energía pasa de "dueño" y, de hecho, este proceso es importante para mantener la vida en el planeta.

La energía en los seres vivos, pareciera que sólo trata de mantenerse en estado activo para darle continuidad a otros seres que están interaccionando sin importarle que unos se desprendan.

\section{Materiales y método}

Contraste interdisciplinario entre el modelo teórico de Sigmund Freud y los aportes actuales de la biología, particularmente desde la fisiología y la ecología. A partir del cotejo entre textos explícitos y significativos sobre el tema abordado en el modelo de Freud y publicaciones recientes en biología.

\section{Resultados}

En la teoría de Freud, la pulsión:

1. Tiene reconocido un carácter biológico.

2. Es considerada como un invariante biológico sin ser susceptible de modificación.

3. Proviene del interior del cuerpo.

4. Debido a su pretendida naturaleza biológica es responsable de la autodestrucción humana.

Desde la biología:

1. La naturaleza es una noción que no posee una sola definición; sin embargo, está asociada con la vida.

2. La energía se involucra con la manutención de diferentes niveles de la vida.

3. La pulsión, como es planteada en la teoría de Freud, no es reconocida como un objeto de estudio para la biología.

\section{Agradecimiento}

Agradecemos a la Universidad Tecnología de México por su apoyo al desarrollo del presente trabajo de investigación.

\section{Conclusiones}

La pulsión (trieb) no es un objeto de estudio de la biología ni de la neurociencia (Yovell, 2016; Yovell, Solms, \& Fotopoulou, 2015) sino a lo sumo, desde el neuropsicoanálisis, reconocida como una hipótesis auxiliar para explicar la motivación en procesos mentales. Asumiendo que las pulsiones representen las interacciones de las vías metabólicas y endocrinológicas (Solms \& Zellner, 2012). Para el neuropsicoanálisis la hipótesis de las pulsiones representa estas interacciones pero no son resultado de ellas. Por lo tanto las pulsiones -del modelo teórico de Freud- son consideradas un recurso expositivo y no explicativo.

El uso del término pulsión, al carecer de fundamento biológico, requiere de otro fundamento disciplinario para contar con valor explicativo. De esta manera su transformación conceptual influirá sobre el abordaje y resolución del sufrimiento que aborda el psicoanálisis.

\section{Referencias}

Bachelard, G. (2010). La formación del espíritu científico: Contribución a un psicoanálisis del conocimiento objetivo. México: Siglo XXI.

Badiou, A. (2009). El concepto de modelo: Introducción a una epistemología materialista de las matemáticas. Buenos Aires: La Bestia Equilátera.

Ballantyne, N. (2019). Knowing Our Limits. Oxford University Press.

Corella, D., \& Ordovas, J. M. (2017). Conceptos básicos en biología molecular relacionados con la genética y la epigenética. Revista Española de Cardiología, 70(9), 744-753.

De la Garza Toledo, E. (2018). Subjetividad, cultura y estructura. Iztapalapa(50), 83-104.

Descola, P. (2012). Más allá de naturaleza y cultura. Buenos Aires: Amorrortu.

Eidelsztein, A. (2008). Por un psicoanálisis no extraterritorial. El Rey está desnudo. Revista del psicoanálisis por venir, 1(1), 66. 
Freud, S. (2010a). Mis tesis sobre el papel de la sexualidad en la etiología de las neurosis (1906 [1905]) (Vol. VII). Buenos Aires: Amorrortu.

Freud, S. (2010b). Pulsiones y destinos de pulsión (1915) (Vol. XIV). Buenos Aires: Amorrortu.

Freud, S. (2010c). Tres ensayos de teoría sexual (1905) (Vol. VII). Buenos Aires: Amorrortu.

Freud, S. (2015). El malestar en la cultura (1930 [1929]) (Vol. XXI). Buenos Aires: Amorrortu.

Freud, S. (2016a). $31^{\circ}$ conferencia. La descomposición de la personalidad psíquica (Vol. XXII). Buenos Aires: Amorrortu.

Freud, S. (2016b). $33^{\circ}$ conferencia. La feminidad (Vol. XXII). Buenos Aires: Amorrortu.

Freud, S. (2016c). Algunas consecuencias psíquicas de la diferencia anatómica entre los sexos (1925) (Vol. XIX). Buenos Aires: Amorrortu.

Freud, S. (2016d). El porvenir de una ilusión (1927) (Vol. XXI). Buenos Aires: Amorrortu.

Freud, S. (2016e). El yo y el ello (1923). Buenos Aires: Amorrortu.

Freud, S. (2016f). Esquema del psicoanálisis (1940 [1938]) (Vol. XXIII). Buenos Aires: Amorrortu.

Freud, S. (2016g). Más allá del principio de placer (1920) (Vol. XVIII). Buenos Aires: Amorrortu.

Freud, S. (2016h). Presentación autobiográfica (1925 [1924]). Buenos Aires: Amorrortu.

Freud, S. (2016i). Psicología de las masas y análisis del yo (1921) (Vol. XVIII). Buenos Aires: Amorrortu.

Freud, S. (2016j). ¿Pueden los legos ejercer el análisis? Diálogos con un juez imparcial (1926) (Vol. XX). Buenos Aires: Amorrortu.

Geertz, C. (2003). La interpretación de las culturas. España: Gedisa.
Kandel, E. R. (2007). En busca de la memoria: Nacimiento de una nueva ciencia de la mente. Buenos Aires: Katz.

Klier, G. (2016). La naturaleza que se conserva: Una aproximación al concepto de biodiversidad. Apuntes de Investigación del CECYP, (27), 207217.

Koyré, A. (1994). Pensar la ciencia (A. B. Mari, Trans.). España: Paidós.

Koyré, A. (2007). Estudios de historia del pensamiento científico. México: Siglo XXI.

Kreiss, D. (2019). The fragmenting of the civil sphere: How partisan identity shapes the moral evaluation of candidates and epistemology. In Politics of meaning/meaning of politics (pp. 223241). Palgrave Macmillan, Cham.

Krymkiewicz, M. (2015). Modelos energéticos en Psicoanálisis. Diferencias entre Sigmund Freud y Jacques Lacan.(Energetic Models in Psychoanalysis. Differences between Sigmund Freud and Jacques Lacan). Affectio Societatis, 12(22), 59-70.

Lakatos, I. (2007). Escritos Filosóficos, 1: La metodología de los programas de investigación científica (J. C. Zapatero, Trans.). España: Alianza Editorial.

Macalady, J. L., Hamilton, T. L., Grettenberger, C. L., Jones, D. S., Tsao, L. E., \& Burgos, W. D. (2013). Energy, ecology and the distribution of microbial life. Philosophical Transactions of the Royal Society B: Biological Sciences, 368(1622), 20120383.

Margulis, L., \& Sagan, D. (1996). ¿Qué es la vida? Nevraumont Publishing Co., Nueva York.

Robles, R. G., Ramírez, P. A. A., \& Velásquez, S. P. P. (2012). Epigenética: definición, bases moleculares e implicaciones en la salud y en la evolución humana. Revista Ciencias de la Salud, 10(1), 59-71.

Schrödinger, E. (2015). What is life? The physical aspect of the living cell and mind. Cambridge: Cambridge University Press. 
Solms, M., \& Zellner, M. R. (2012). Freudian drive theory today. In A. Fotopoulou, D. Pfaff, \& M. A. Conway (Eds.), From the couch to the lab: Trends in psychodynamic neuroscience (pp. 49-63). NY: Oxford University Press New York.

Subaş1, A. (2019). A Dynamic Systems Theory of epistemic curiosity. New Ideas in Psychology, $54,8-14$.

Swanson, D. R. (1977). A critique of psychic energy as an explanatory concept. Journal of the American Psychoanalytic Association, 25(3), 603-633.

Torri, P. (2019). The telos of Assimilation to God and the Conflict between theoria and praxis in Plato and the Middle Platonists. en Thinking, Knowing, Acting: Epistemology and Ethics in Plato and Ancient Platonism (pp. 228-250). Brill.

Van Damme, M. J., Anseel, F., Duyck, W., \& Rietzschel, E. F. (2019). Strategies to improve selection of creative ideas: An experimental test of epistemic and social motivation in groups. Creativity and Innovation Management, 28(1), 61-71.

Van Dijk, T. A. (1999). El análisis crítico del discurso. Revista anthropos: Huellas del conocimiento(186), 23-36.

Van Dijk, T. A. (2012). Estructuras y funciones del discurso: una introducción interdisciplinaria a la lingüística del texto y a los estudios del discurso. México: Siglo XXI.

Virno, P. (2005). Cuando el verbo se hace carne: Lenguaje y naturaleza humana. Madrid: Traficantes de Sueños.

Yovell, Y. (2016). Drive and Love: Revisiting Freud's Drive Theory. In A NeuroPsychoanalytical Dialogue for Bridging Freud and the Neurosciences (pp. 127-136): Springer.

Yovell, Y., Solms, M., \& Fotopoulou, A. (2015). The case for neuropsychoanalysis: Why a dialogue with neuroscience is necessary but not sufficient for psychoanalysis. The International Journal of Psychoanalysis. 